\title{
La relación entre la formación superior y la inserción profesional en países latinoamericanos ${ }^{1}$
}

The relation between higher education and professional insertion in Latin American countries

Dr. Eduardo Chávez Molina

Investigador - Instituto de Investigaciones Gino Germani (IIGG)

Universidad de Buenos Aires (UBA). Profesor y Director del Departamento de Sociología, Universidad Nacional de Mar del Plata.

Email:echavez@sociales.uba.ar

\section{Mg. María Clara Fernández Melián}

Auxiliar de investigación - IIGG, UBA, Argentina

Profesora - Universidad Nacional de Tres de Febrero

Email: mfernandezmelian@sociales.uba.ar

\begin{abstract}
Resumen: El artículo tiene como propósito analizar la situación del empleo de los profesionales con nivel educativo superior en distintos países de América Latina, observando específicamente las distancias y correspondencias existentes entre las credenciales educativas y la calificación del empleo. Nuestra observación pone el acento en este punto, ¿cuán posible es obtener una inserción profesional adecuada de los egresados de la educación superior según los últimos datos existentes en Latinoamérica considerando el contexto de desarrollo de cada uno de los países seleccionados? En esa línea, los interrogantes que nos orientan son: ¿la inserción ocupacional de los universitarios es parecida entre varones y mujeres? A su vez, la edad también aparece como una variable sintomática de las experiencias, habilidades, y de la consolidación de los estudios formales; la forma de consolidación laboral implica claras diferencias respecto del tipo de inserción y resultados económicos de acuerdo con la edad del
\end{abstract}

\footnotetext{
${ }^{1}$ Este artículo se encuentra inserto en la dimensión internacional del Proyecto Coordinado DINAMOS: DINAMOS1 (PID2019-106548GB-C21) "Movilidad social intrageneracional y trayectorias ocupacionales segmentadas" y DINAMOS2 (PID2019-106548GA-C22) "Movilidad social intergeneracional: la educación como mecanismo explicativo", Ministerio de Ciencia e Innovación, Generación de Conocimiento y Fortalecimiento científico y tecnológico del Sistema de I+D+i e I+D+i orientado a los Retos de la Sociedad 2019 (https://pagines.uab.cat/dinamos/).
} 
ocupado/a. Por último, nos preguntamos: ¿en qué ramas u ocupaciones se observan las mayores similitudes y divergencias? Para ello, utilizamos los datos de la Encuesta Latinobarómetro de Argentina, Bolivia, Brasil, Chile, Colombia, Costa Rica, Rep. Dominicana, Ecuador, El Salvador, Guatemala, Honduras, México, Nicaragua, Panamá, Paraguay, Perú, Uruguay y Venezuela. Así como también la información proveniente del Índice de Desarrollo Humano de Naciones Unidas, para dar el contexto en el cual se desarrolla dicha inserción en cada país.

Palabras clave: credenciales educativas, calificación ocupacional, inserción profesional, educación superior, América Latina

Abstract: The purpose of the article is to analyse the employment situation of professionals with a higher education level in different Latin American countries, specifically observing the distances and correspondences between educational credentials and job qualifications. Our observation puts the accent on this point, how possible is it to obtain an adequate professional insertion of graduates of higher education according to the latest data in Latin America considering the development context of each of the selected countries?

Along these lines, the questions that guide us are: is it the occupational insertion of university students similar between men and women? In turn, age also appears as a symptomatic variable of experiences, abilities, and the consolidation of formal studies. The form of labour consolidation implies clear differences regarding the type of insertion and economic results according to the age of the employed person. Finally, we ask: in which branches or occupations are the greatest similarities and divergences observed?

For this, we use data collected by Latinobarómetro Survey from Argentina, Bolivia, Brazil, Chile, Colombia, Costa Rica, Dominican Republic, Ecuador, El Salvador, Guatemala, Honduras, Mexico, Nicaragua, Panamá, Paraguay, Perú, Uruguay and Venezuela. As well as the information from the United Nations Human Development Index, to give the context in which this insertion is developed in each country.

Keywords: educational credentials, occupational qualification, professional insertion, higher education, Latin America 


\section{Introducción y contexto de la investigación}

De acuerdo con los estudios que se vinculan a esta temática, hay cierto consenso sobre el aporte de la educación superior al desarrollo económico de América Latina, en el sentido de la incidencia en la innovación y la ayuda potencial en fortalecer la competitividad internacional de la estructura productiva, como así también en "contribuir a una mayor expansión económica a largo plazo" (Moreno-Bridy RuizNápoles, 2009: 5).

Ciertamente, en la región las universidades públicas, sobre todo, son las que mantienen el avance de la ciencia y la tecnología, sin este conocimiento sería altamente improbable que la región alcance un desarrollo económico adecuado.

Además, como expresión de comportamientos societales, asistimos a "un crecimiento continuado de los niveles educativos de la población, siendo cada generación más educada que la precedente, crecimiento que, en las últimas décadas, se ha basado esencialmente en el aumento del nivel educativo superior, y en mayor proporción de las mujeres" tanto en asistencia a la universidad como en el propio egreso (Beduwe y Germe, 2004, Planas y Fachelli, 2016). Se han implementado diversos tipos de políticas para mejorar el acceso, la permanencia y/o la finalización en el sistema educativo de nivel superior como resultado de tradiciones institucionales y educativas particulares en cada uno de los países latinoamericanos (Unzué, 2012) que han colaborado fuertemente en este avance. Específicamente desde mediados del siglo XX en adelante, se produce cierta masificación de la matrícula en la educación superior, así como también crece el número de establecimientos $\mathrm{y}$, por ende, se expande también su cuerpo docente (Brunner, 1990).

Si bien las recurrentes crisis económicas y sociales de la región han impactado en la posibilidad de profundizar de forma sostenida estas mejoras, la esperanza puesta en un porvenir mejor para los jóvenes, vía la educación superior parece mostrar aún señales saludables para que dicha "promesa" se cumpla, tomando la evidencia de estudios anteriores. Alcanzar estudios superiores implica en muchos casos, la garantía de logros para acceder a condiciones de vida aceptable, tanto en términos de inserción adecuada al mundo del trabajo, como la obtención de recursos suficientes para que dichos logros se materialicen en recursos (Chávez Molina, 2019). 
Siguiendo esta línea, es necesario pensar el vínculo entre educación y empleo y el grado de congruencia entre ellos. La inserción adecuada está pensada en la lógica de que la ocupación sea acorde con los estudios educativos y sistemáticos realizados, y en correspondencia, los ingresos tengan vinculación con la calificación de la tarea laboral. Nuestra aproximación a la "inserción adecuada", será el vínculo entre el nivel educativo profesional y la calificación en la tarea.

En los estudios tradicionales de movilidad social, un factor adscriptivo preponderante siempre ha sido, en la región, la educación, en el sentido de que las posibilidades de mayor nivel educativo proveen una mayor chance de ascenso social, y mayor bienestar. La educación, y sobre todo la educación superior aparece ligada a la mayor probabilidad de ascenso social, y, por ende, a un bienestar material; emerge como una promesa cierta, incentivada además por una mayor diversificación y calificación de los puestos a ocupar en las sociedades modernas, tanto por una mayor demanda de especificidad profesional en los empleos, como la oferta más calificada de las generaciones jóvenes en el país (Rama, 2009).

A su vez, existe consenso que en las sociedades modernas se ha incrementado el rol mediador de la educación entre orígenes y destinos de clase, pero este mecanismo está asociado a la reproducción intergeneracional de las desigualdades. Para que la educación se transforme en una canal de igualación de oportunidades y de movilidad social ascendente para los grupos más desfavorecidos es necesario que se cumplan al menos una de la siguientes condiciones: ecualización, que implica que disminuya el efecto del origen de clase social sobre el nivel educativo alcanzado y que se incrementen los retornos del aumento de las credenciales educativas, aumentando su peso en el destino de clase; y/o atenuación, que implica que la asociación entre orígenes y destinos de clase se debilite con el incremento en la escolaridad ( Solís y Dalle, 2019).

Asimismo, el nivel de desarrollo de cada país constituye un factor condicionante de relevancia. Si bien existen diversas teorías al respecto En ese sentido, rescatamos el trabajo de Satrústegui quien plantea que a lo "largo de las últimas décadas, la economía del desarrollo y, más en general, los estudios sobre desarrollo -entendidos de manera amplia como el análisis de las condiciones capaces de favorecer el progreso y el bienestar humanos- atraviesan por una cierta crisis. Frente al vigor y la relevancia de los debates habidos durante la segunda mitad del siglo XX, pareciera que en la actualidad los estudios sobre desarrollo han ido perdiendo importancia en el ámbito de las ciencias 
sociales, en favor de enfoques centrados en el corto plazo y/o en el análisis coyuntural de realidades particulares”. (Satrústegui, K. U.; 2009). Y con la intención de cuantificar el contexto en el cual se desenvuelve el proceso de la educación superior, visualizaremos la situación del Índice de Desarrollo Humano (IDH) de cada país, y su situación en torno a la educación superior. De esta manera, se brindan algunas pistas para reflexionar sobre los matices al interior de la región en torno a la correspondencia entre el acceso a la educación superior y el tipo de actividad laboral que se desempeña.

\section{Consideraciones metodológicas}

Se trabaja con la Encuesta Latinobarómetro más reciente, correspondiente al año 2018 porque permite realizar un análisis comparativo amplio que contempla diversos países de América Latina y el Caribe, con el Índice de Desarrollo Humano elaborado por la Organización de Naciones Unidas del año 2019 (publicado en 2020) en tanto permite analizar los resultados de forma contextual y con estadísticas sobre la situación de pobreza y sobre el mercado laboral, proveniente del Centro de Estudios Distributivos, Laborales y Sociales (CEDLAS).

Para la operacionalización de las variables de la Encuesta Latinobarómetro se tomaron algunas decisiones fundadas en la disponibilidad de los datos de la fuente seleccionada y en la literatura existente. Cabe destacar que la encuesta realizada por Latinobarómetro no incluye la ocupación desglosada en un clasificador, sino que se cuenta con información mucho más agregada, motivo por el cual se detalla a continuación su tratamiento. Se toma al universo de población de 30 años y más en general, y en el caso de la adecuación laboral, se considera a quienes tienen entre 30 y 65 años porque son quienes se encuentran en una situación de madurez ocupacional, es decir, que han atravesado la mayor parte de su carrera laboral, logrando consolidar su inserción o estando próximos a hacerlo (Echeverría Zabalza, 1999; Erikson y Goldthorpe, 1992).

La variable sector de actividad será reclasificada a los fines de esta investigación de la siguiente manera ${ }^{2}:$ 1) sector primario que incluye agricultura, ganadería, caza, silvicultura y pesca, y explotación de minas y canteras, 2) sector secundario formado por

\footnotetext{
${ }^{2}$ Si bien existen esquemas más recientes que distinguen al sector cuaternario como aquel formado por industrias relativas a los servicios de información por una cuestión de disponibilidad de datos en la fuente que utilizamos sólo es posible trabajar con la división tradicional de tres sectores.
} 
industrias manufactureras, y 3) sector terciario que contiene servicios y administración pública y defensa.

Por otra parte, utilizaremos la variable tipo de trabajo como indicador proxy de la calificación ocupacional del empleo, y la reagruparemos en dos categorías, siguiendo el trabajo anterior de Chávez Molina (2019): 1) Profesional Calificado donde se encuentran profesionales, dueños de negocios, profesionales, altos ejecutivos, ejecutivos de mando medio, y 2) Trabajadores no calificados en donde se aglutinan agricultores/pescadores, trabajadores por cuenta propia/ambulante y otros empleados. A su vez, se trabaja con el nivel socioeconómico cuyas categorías fueron agrupadas quedando los siguientes valores: 1) muy bueno o bueno, 2) regular, 3) malo o muy malo ${ }^{3}$.

El nivel educativo consta de las siguientes categorías: 1) básica y menos, 2) secundaria, media, técnica y menos, y 3) superior o más.

Los países fueron agrupados en base a las mayores similitudes y divergencias respecto de la situación educativa y laboral: Cono sur (Argentina, Brasil, Chile, Paraguay, Uruguay y Brasil), Centroamérica y el Caribe (Costa Rica, República Dominicana, El Salvador, Guatemala, Honduras, México, Nicaragua, y Panamá) y Países andinos (Bolivia, Colombia, Venezuela, Ecuador y Perú).

Por último, la variable sobre adecuación profesional se construye a partir de la concordancia entre el nivel educativo superior o más y la calificación ocupacional profesional.

\section{Análisis}

Cuando se toma al conjunto de los países de América Latina se observa una disminución de la proporción de población a medida que se asciende de nivel educativo (Cuadro 1): casi la mitad de la población de la región tiene un nivel educativo básico, un poco más de un tercio accede a nivel medio y un $20 \%$ concluye nivel superior. Sin embargo, hacia el interior se observan fuertes diferencias. Centroamérica y el Caribe tienen la menor proporción de población con nivel educativo superior, siendo del 14\%, mientras que los

\footnotetext{
${ }^{3}$ Cabe destacar que el nivel socioeconómico se obtiene a partir de la apreciación por parte del entrevistador tomando como punto de referencia: la calidad de la vivienda, calidad del amueblado y la apariencia general del encuestado.
} 
países del Cono sur están en una situación intermedia con un $21 \%$ y los países andinos exhiben el mayor porcentaje alcanzando un $28 \% 4$.

De todas maneras, las diferencias no solo se encuentran entre las regiones sino al interior de estas. En el cono sur, Chile se destaca por contar con el mayor nivel de egresados de nivel superior, siendo de más del 30\%. Pero dentro de esta misma región se encuentra Paraguay con menos del 10\% de su población en esa situación. En Centroamérica y el Caribe el caso de Honduras se destaca por estar muy por debajo de la media de su región, con sólo un $4 \%$ de población con nivel superior o más; mientras que entre los países andinos las diferencias son más reducidas, y no llegan a ser mayores a ocho puntos.

A su vez, al hacer foco en el nivel superior podemos identificar países con niveles similares pero que se encuentran dentro de diferentes regiones: los mayores valores se encuentran en Venezuela, Ecuador, Perú y Chile con entre un $30 \%$ y 35\%, mientras que Uruguay, Colombia, Costa Rica, Argentina, República Dominicana, Panamá, Bolivia y Brasil oscilan alrededor de un $20 \%$, y los siguientes países se encuentran bastante por debajo de esa situación, con un 10\% o menos: México, El Salvador, Paraguay, Nicaragua, Guatemala y Honduras.

\footnotetext{
${ }^{4}$ El nivel educativo superior de la población adulta presentado en el artículo consta de los datos obtenidos de Latinobarómetro, que presentan ciertas diferencias de la información brindada por SITEAL/UNESCO, que se puede consultar en el siguiente enlace: https://siteal.iiep.unesco.org/indicadores. Cabe mencionar que estas disparidades podrían deberse a los criterios utilizados en los datos obtenidos de Latinobarómetro para definir el nivel superior en cada país. En algunos países la educación técnica puede corresponder a nivel secundario y en otros a nivel superior y, además, se observa que los años de escolaridad para alcanzar el nivel superior difieren entre los distintos países. Para más información consultar el informe metodológico 2018 en: https://www.latinobarometro.org/latContents.jsp
} 
Cuadro 1. Porcentaje de población de 30 años o más según nivel educativo y país/región. América Latina y el Caribe, 2018

\begin{tabular}{|c|c|c|c|c|}
\hline \multirow[b]{2}{*}{ Región/País } & \multicolumn{3}{|c|}{ Nivel educativo } & \multirow[b]{2}{*}{ Total } \\
\hline & $\begin{array}{c}\text { Básica y } \\
\text { menos }\end{array}$ & $\begin{array}{l}\text { Secundaria, } \\
\text { media, } \\
\text { técnica y } \\
\text { menos }\end{array}$ & $\begin{array}{l}\text { Superior o } \\
\text { más }\end{array}$ & \\
\hline Cono sur & 42,2 & 37,1 & 20,7 & 100 \\
\hline Chile & 10,7 & 58,7 & 30,7 & 100 \\
\hline Uruguay & 35,8 & 41,0 & 23,2 & 100 \\
\hline Argentina & 48,8 & 29,4 & 21,8 & 100 \\
\hline Brasil & 43,7 & 38,2 & 18,2 & 100 \\
\hline Paraguay & 73,7 & 17,3 & 9,0 & 100 \\
\hline Centroamérica y el Caribe & 54,3 & 32,0 & 13,7 & 100 \\
\hline Costa Rica & 53,2 & 24,9 & 21,9 & 100 \\
\hline República Dominicana & 42,0 & 36,9 & 21,1 & 100 \\
\hline Panamá & 40,9 & 38,1 & 21,0 & 100 \\
\hline México & 47,2 & 39,7 & 13,1 & 100 \\
\hline El Salvador & 36,7 & 51,1 & 12,1 & 100 \\
\hline Nicaragua & 68,5 & 23,3 & 8,2 & 100 \\
\hline Guatemala & 73,8 & 20,3 & 5,9 & 100 \\
\hline Honduras & 79,2 & 17,0 & 3,8 & 100 \\
\hline Países andinos & 36,9 & 35,2 & 27,8 & 100 \\
\hline Venezuela & 22,1 & 42,5 & 35,4 & 100 \\
\hline Ecuador & 28,5 & 40,3 & 31,2 & 100 \\
\hline Perú & 39,1 & 30,8 & 30,1 & 100 \\
\hline Colombia & 45,3 & 32,4 & 22,3 & 100 \\
\hline Bolivia & 51,0 & 29,5 & 19,6 & 100 \\
\hline Total América Latina & 45,3 & 34,6 & 20,1 & 100 \\
\hline
\end{tabular}

Fuente: elaboración propia en base a Latinobarómetro, 2018.

Por otro lado, el panorama social de los países seleccionados es diverso, como puede comprobarse en el gráfico 1, aunque hay correspondencia entre aquellos países con niveles más altos de pobreza por USD calculados según Poder de Paridad Adquisitivo PPA- (Línea de Pobreza, menos de 5,5 dólares por día, base 2011) y mayores niveles de desigualdad de ingresos según el índice de concentración GINI, como Guatemala y Honduras; otros como Uruguay presentan baja cantidad de personas bajo la línea de pobreza, y bajo nivel de desigualdad, Chile presenta también valores bajos de pobreza, pero presenta una peor distribución del ingreso. En un escalón de indicadores más bajo se encuentran Argentina, Panamá y Costa Rica, con valores similares, aunque es Argentina el que presenta un Gini menor. 
Por otro lado, lo que queda claro en el cuadro, es que no hay una vinculación directa entre pobreza y desocupación, ya que el factor de escasez de ingresos en América Latina está más ligado a empleos desprotegidos, o empleos formales de bajos ingresos, es decir, a situaciones de pauperización laboral.

Gráfico 1. Tasa de desempleo, pobreza según USD PPA y Coeficiente de Gini por país. América Latina y el Caribe.

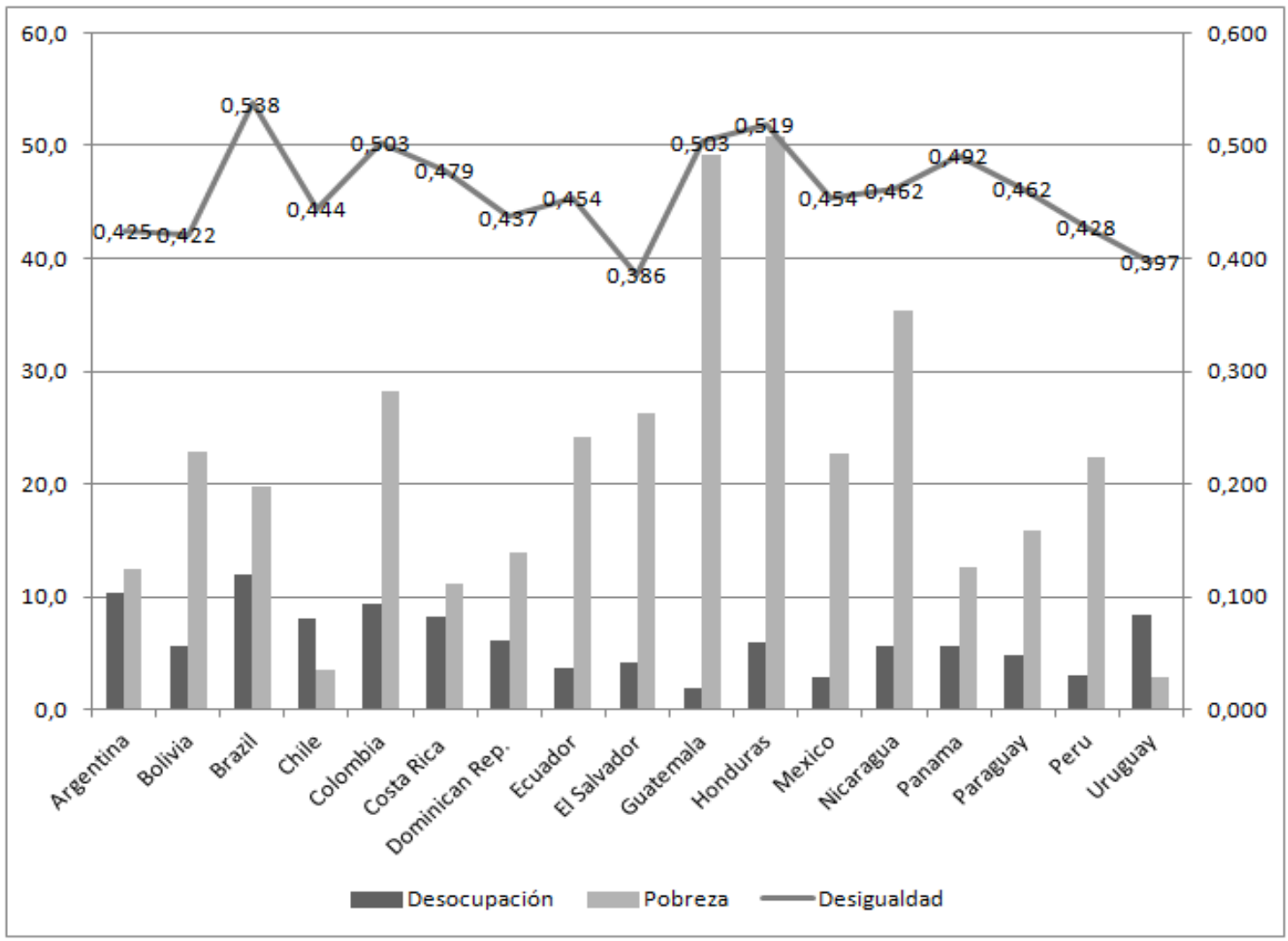

Fuente: elaboración propia en base a estadísticas CEDLAS para años 2014-2019

Presentamos en el cuadro 2 el IDH de América Latina, replicando la clasificación del PNUD (Programa de las Naciones Unidas para el Desarrollo). Entre países con IDH muy alto se encuentran Chile, Argentina y Uruguay, y luego Panamá y Costa Rica. Luego la clasificación replica el IDH Alto, Medio, y Bajo, donde se sitúan el resto de los países. Hay coincidencia general, entre los países con IDH elevado y mayor proporción de población con educación superior. 


\section{Cuadro 2. Índice de Desarrollo Humano según país. América Latina y el Caribe.}

Tabla 1 Índice de Desarrollo Humano

\begin{tabular}{|l|l|c|c|}
\hline \multicolumn{5}{|c|}{ IDH Muy alto } \\
\hline Puesto & País & IDH según datos del 2019 & Puesto en el mundo \\
\hline 1 & Chile & 0,851 & 43 \\
\hline 2 & Argentina & 0,845 & 46 \\
\hline 3 & Uruguay & 0,817 & 55 \\
\hline 4 & Panamá & 0,815 & 57 \\
\hline 5 & Costa Rica & 0,810 & 62 \\
\hline \multicolumn{5}{|c|}{ IDH Alto } \\
\hline 6 & Cuba & 0,783 & 70 \\
\hline 7 & México & 0,779 & 74 \\
\hline 8 & Perú & 0,777 & 79 \\
\hline 9 & Colombia & 0,767 & 83 \\
\hline 10 & Brasil & 0,765 & 84 \\
\hline 11 & Ecuador & 0,759 & 88 \\
\hline 12 & República Dominicana & 0,756 & 103 \\
\hline 13 & Paraguay & 0,728 & 107 \\
\hline 14 & Bolivia & 0,718 & 113 \\
\hline 15 & Venezuela & 0,711 & 124 \\
\hline \multicolumn{5}{|l|}{} & IDH Medio & 127 \\
\hline 16 & El Salvador & 0,673 & 128 \\
\hline 17 & Guatemala & 0,663 & 132 \\
\hline 18 & Nicaragua & 0,660 & 170 \\
\hline 19 & Honduras & 0,634 & \\
\hline \multicolumn{7}{|l|}{} & 0,510 & \\
\hline 20 & Haití & & \\
\hline
\end{tabular}

Fuente: PNUD 2020

El cuadro 3 presenta la correspondencia entre el nivel educativo y la calificación ocupacional, introduciendo la preocupación principal de este trabajo. En primer lugar, vemos que alrededor de un $60 \%$ de las personas de nivel educativo superior desempeñan ocupaciones calificadas, siendo levemente mayor esta proporción en Centroamérica y el Caribe en comparación con los países andinos y los del Cono sur (Cuadro 3). Así, en definitiva, se logra dimensionar la problemática de que 4 de cada 10 egresados del nivel superior no logran ubicarse en puestos de calificación acorde a sus credenciales educativas.

Por otro lado, el acceso a puestos calificados se reduce significativamente para quienes poseen credenciales básicas o medias, siendo entre un $20 \%$ y un $30 \%$ de los casos. 
Cuadro 3. Porcentaje de población de 30 años y más según calificación ocupacional, región y nivel educativo. América Latina y el Caribe, 2018.

\begin{tabular}{|c|c|c|c|c|}
\hline \multirow[b]{2}{*}{ Región } & \multirow[b]{2}{*}{$\begin{array}{l}\text { Calificación } \\
\text { ocupacional }\end{array}$} & \multicolumn{3}{|c|}{ Nivel educativo } \\
\hline & & $\begin{array}{c}\text { Básica y } \\
\text { menos }\end{array}$ & $\begin{array}{l}\text { Secundaria, } \\
\text { media, técnica } \\
\text { y menos }\end{array}$ & $\begin{array}{l}\text { Superior o } \\
\text { más }\end{array}$ \\
\hline \multirow{3}{*}{ Cono sur } & Calificado profesional & 16,7 & 21,3 & 60,6 \\
\hline & No calificado & 83,3 & 78,7 & 39,4 \\
\hline & Total & 100 & 100 & 100 \\
\hline \multirow{3}{*}{$\begin{array}{c}\text { Centroamérica y } \\
\text { el Caribe }\end{array}$} & Calificado profesional & 20,6 & 30,6 & 64,6 \\
\hline & No calificado & 79,4 & 69,4 & 35,4 \\
\hline & Total & 100 & 100 & 100 \\
\hline \multirow{3}{*}{ Países andinos } & Calificado profesional & 21,5 & 24,9 & 62,3 \\
\hline & No calificado & 78,5 & 75,1 & 37,7 \\
\hline & Total & 100 & 100 & 100 \\
\hline \multirow{3}{*}{ América Latina } & Calificado profesional & 19,8 & 25,7 & 62,3 \\
\hline & No calificado & 80,2 & 74,3 & 37,7 \\
\hline & Total & 100 & 100 & 100 \\
\hline
\end{tabular}

Fuente: elaboración propia en base a Latinobarómetro, 2018.

Cuando nos adentramos en la desagregación de la correspondencia entre nivel educativo y calificación ocupacional según sexo para el total de la Región no se observan diferencias en el caso de los estudios superiores, aunque sí en los estudios medios y bajos (Cuadro 4). En esta línea, las mujeres de niveles educativos básicos y medios logran acceder a puestos calificados en mayor medida que los varones, aunque la diferencia es leve, siendo entre 6 y 3 puntos porcentuales respectivamente.

Sin embargo, al distinguir al interior de la Región, surgen algunos matices: en Centroamérica y el Caribe las mujeres con educación superior en empleos calificados ascienden a $66 \%$ versus el $63 \%$ en el caso de los varones. A su vez, las mujeres con nivel educativo básico o menos ocupan posiciones profesionales en mayor medida que los varones tanto en el Cono sur como en los países andinos (entre 10 y 8 puntos respectivamente), lo que no se observa para Centroamérica y el Caribe. 
Cuadro 4. Porcentaje de población de nivel educativo profesional de 30 años y más según calificación ocupacional, región y sexo. América Latina y el Caribe, 2018.

\begin{tabular}{|c|c|c|c|c|c|}
\hline \multirow[b]{2}{*}{ Región } & \multirow[b]{2}{*}{ Sexo } & \multirow[b]{2}{*}{$\begin{array}{l}\text { Calificación } \\
\text { ocupacional }\end{array}$} & \multicolumn{3}{|c|}{ Nivel educativo } \\
\hline & & & $\begin{array}{c}\text { Básica y } \\
\text { menos }\end{array}$ & $\begin{array}{l}\text { Secundaria, } \\
\text { media, } \\
\text { técnica y } \\
\text { menos }\end{array}$ & $\begin{array}{c}\text { Superior } \\
\text { o más }\end{array}$ \\
\hline \multirow{6}{*}{ Cono sur } & \multirow{3}{*}{ Varón } & Calificado profesional & 13,3 & 19,7 & 61,1 \\
\hline & & No calificado & 86,7 & 80,3 & 38,9 \\
\hline & & Total & 100 & 100 & 100 \\
\hline & \multirow{3}{*}{ Mujer } & Calificado profesional & 22,3 & 23,7 & 60,1 \\
\hline & & No calificado & 77,7 & 76,3 & 39,9 \\
\hline & & Total & 100 & 100 & 100 \\
\hline \multirow{6}{*}{$\begin{array}{l}\text { Centroaméric } \\
\text { a y el Caribe }\end{array}$} & \multirow{3}{*}{ Varón } & Calificado profesional & 19,2 & 31,0 & 63,2 \\
\hline & & No calificado & 80,8 & 69,0 & 36,8 \\
\hline & & Total & 100 & 100 & 100 \\
\hline & \multirow{3}{*}{ Mujer } & Calificado profesional & 23,3 & 30,0 & 66,5 \\
\hline & & No calificado & 76,7 & 70,0 & 33,5 \\
\hline & & Total & 100 & 100 & 100 \\
\hline \multirow{6}{*}{$\begin{array}{c}\text { Países } \\
\text { andinos }\end{array}$} & \multirow{3}{*}{ Varón } & Calificado profesional & 18,0 & 23,3 & 62,2 \\
\hline & & No calificado & 82,0 & 76,7 & 37,8 \\
\hline & & Total & 100 & 100 & 100 \\
\hline & \multirow{3}{*}{ Mujer } & Calificado profesional & 26,1 & 27,2 & 62,3 \\
\hline & & No calificado & 73,9 & 72,8 & 37,7 \\
\hline & & Total & 100 & 100 & 100 \\
\hline \multirow{6}{*}{$\begin{array}{c}\text { América } \\
\text { Latina }\end{array}$} & \multirow{3}{*}{ Varón } & Calificado profesional & 17,3 & 24,7 & 62,2 \\
\hline & & No calificado & 82,7 & 75,3 & 37,8 \\
\hline & & Total & 100 & 100 & 100 \\
\hline & \multirow{3}{*}{ Mujer } & Calificado profesional & 23,9 & 27,0 & 62,5 \\
\hline & & No calificado & 76,1 & 73,0 & 37,5 \\
\hline & & Total & 100 & 100 & 100 \\
\hline
\end{tabular}

Fuente: elaboración propia en base a Latinobarómetro, 2018.

Otra cuestión pertinente es analizar lo que sucede con la adecuación laboral según grupos etarios y sexo, y tal como se desprende del gráfico 2 los niveles presentan diferencias relevantes entre varones y mujeres en determinadas edades. Entre los 30 y 35 años las brechas respecto a la adecuación laboral no son tan marcadas entre sexos, siendo $62 \%$ para los varones y 59\% para las mujeres. Pero luego, esta situación se invierte y entre los 36 y 55 años las mujeres alcanzan mayores proporciones de adecuación laboral y los varones mantienen relativamente su nivel, lo que redunda en que ellas logran superar el porcentaje de adecuación respecto a ellos. Por último, entre los 56 y 65 años las brechas vuelven a manifestarse como en las edades más tempranas y los varones ascienden a un $68 \%$ de adecuación ocupacional frente a un $63 \%$ para las mujeres. A grandes rasgos, 
podrían mencionarse dos cuestiones que permitirían explicar estas brechas: por un lado, en las edades más jóvenes la existencia de una mayor carga de tareas reproductivas y de cuidado. Mientras que, por otro lado, en las edades más avanzadas la problemática del techo y paredes de cristal, esto es, las limitaciones de las mujeres en cuanto al acceso a puestos de mayor profesionalización y jerarquía así como a sectores de mayor dinamismo y remuneración, podría ser un factor preponderante.

De todas maneras, estos hallazgos abren la posibilidad de realizar un análisis de tipo longitudinal que permita seguir trayectorias de varones y mujeres para iluminar algunos interrogantes, como por ejemplo si existen diferencias respecto a la adecuación que tengan que ver con las tareas reproductivas y/o de cuidado así como con las discriminaciones laborales en cada rango etario.

\section{Gráfico 2. Porcentaje de adecuación profesional/laboral según grupos etarios y sexo. América Latina y el Caribe, 2018}
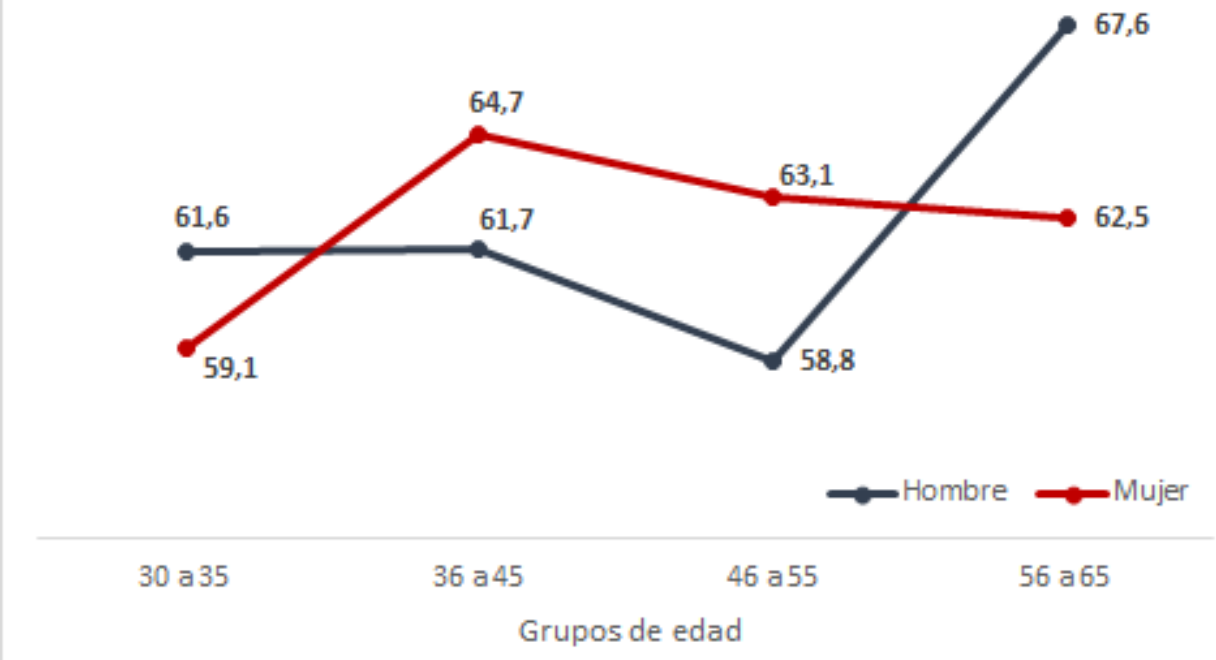

Fuente: elaboración propia en base a Latinobarómetro, 2018.

El gráfico 3 nos muestra la adecuación profesional según la edad y el sector de actividad donde se desempeña la población. Se observa una mayor adecuación en el sector terciario (servicios) que en el primario/secundario (agricultura e industria) para todas las edades, aunque esa brecha es mayor entre quienes tienen 46 a 55 años y 30 a 35 años, siendo el $60 \%$ y $40 \%$ respectivamente. Luego, en las edades más avanzadas, entre 56 a 65 años la proporción de adecuación laboral entre sectores resulta muy similar. 


\section{Gráfico 3. Porcentaje de adecuación profesional/laboral según grupos} etarios y sector de actividad. América Latina y el Caribe, 2018.

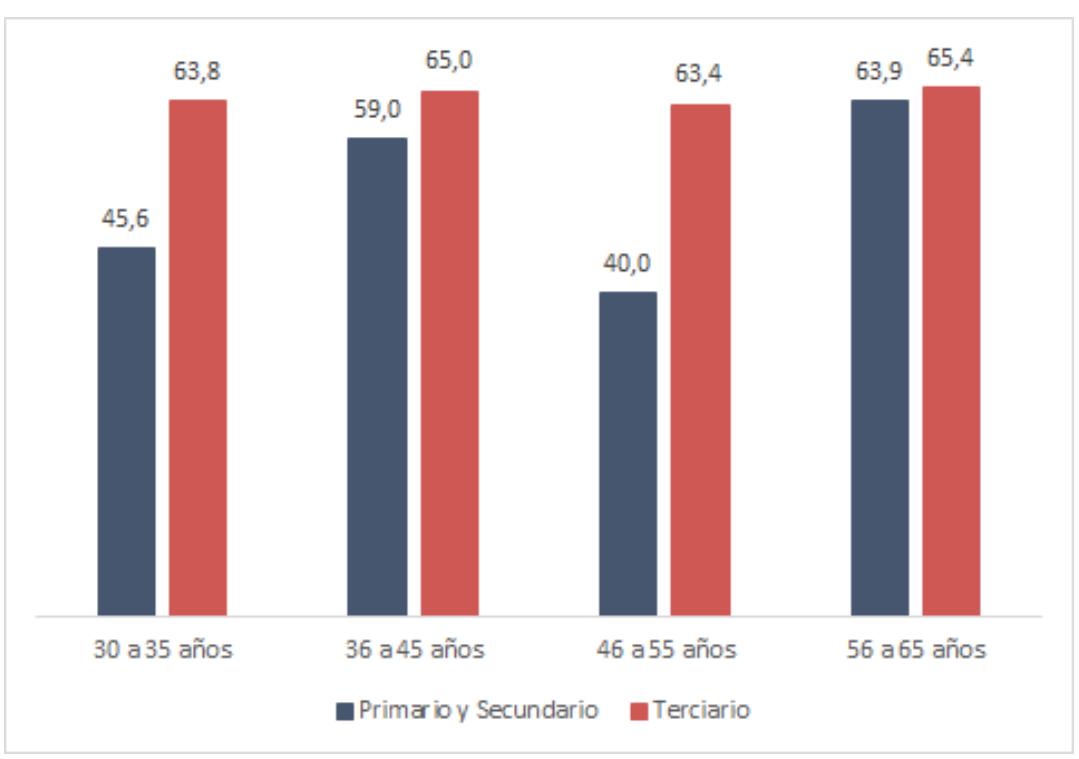

Fuente: elaboración propia en base a Latinobarómetro, 2018.

Por último, la adecuación laboral por grupos etarios y regiones exhibe comportamientos diferenciales: en las edades más jóvenes (de 30 a 35 años) la mayor correspondencia entre nivel educativo superior y ocupación profesional se da en los países andinos, entre quienes tienen de 46 a 55 años la mayor adecuación se da en Centroamérica y el Caribe, y, por último, la población de 56 a 65 años presenta menor adecuación en el Cono sur frente a las restantes regiones.

En general, se observa que los países andinos tienen un alto porcentaje de adecuación puntualmente en las edades más avanzadas, en Centroamérica y el Caribe crece la adecuación conforme lo hace también la edad de la población, y en el Cono sur se mantiene relativamente estable para los diferentes grupos etarios. 


\section{Gráfico 4. Porcentaje de adecuación profesional/laboral según grupos} etarios y región. América Latina y el Caribe, 2018

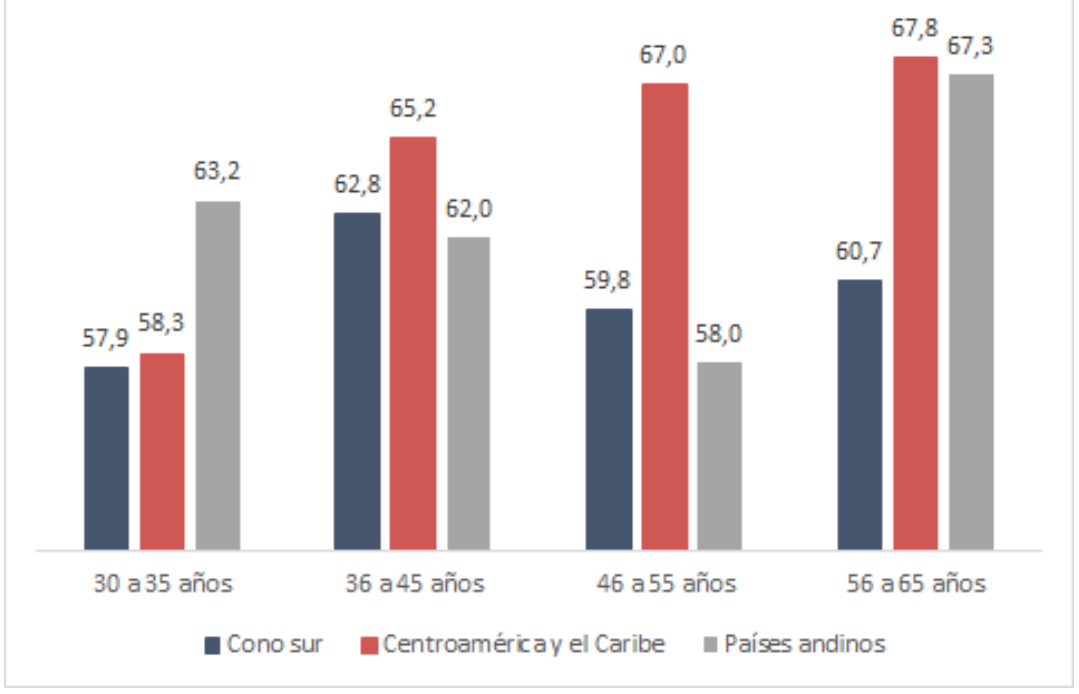

Fuente: elaboración propia en base a Latinobarómetro, 2018.

En última instancia, se revisó el vínculo entre el nivel educativo de la población y el nivel socioeconómico y efectivamente se observa que quienes acceden a nivel superior tienen en mayor medida un nivel socioeconómico muy bueno o bueno, siendo mayor al $70 \%$ (Cuadro 5). Aún más, cuando se puntualiza entre quienes poseen credenciales educativas de nivel superior y además ocupan puestos laborales también calificados la pertenencia al nivel socioeconómico alto es mayor que en los casos en los que no se da esa adecuación profesional, en el primer caso esa proporción llega al 78\% y en el segundo es del $64 \%$ (Cuadro 6).

Cuadro 5. Porcentaje de población de 30 años y más según nivel educativo y socioeconómico. Países de América Latina, 2018

\begin{tabular}{|c|c|c|c|}
\hline \multirow{2}{*}{$\begin{array}{c}\text { Nivel } \\
\text { socioeconómico }\end{array}$} & \multicolumn{3}{|c|}{ Nivel educativo } \\
\cline { 2 - 4 } & Básica y menos & $\begin{array}{c}\text { Secundaria, } \\
\text { media, técnica y } \\
\text { menos }\end{array}$ & Superior o más \\
\hline Muy bueno o bueno & 35,3 & 50,9 & 72,8 \\
\hline Regular & 44,8 & 41,4 & 25,2 \\
\hline Malo o muy malo & 19,9 & 7,7 & 2,0 \\
\hline
\end{tabular}

Fuente: elaboración propia en base a Latinobarómetro, 2018 


\section{Cuadro 6. Porcentaje de población de 30 años y más con nivel} superior completo o más. Países de América Latina, 2018

\begin{tabular}{|c|c|c|}
\hline \multirow{2}{*}{ Nivel socioeconómico } & \multicolumn{2}{|c|}{ Adecuación profesional } \\
\cline { 2 - 3 } & Sí & No \\
\hline Muy bueno o bueno & 78,6 & 64,0 \\
\hline Regular & 20,3 & 33,0 \\
\hline Malo o muy malo & 1,2 & 2,9 \\
\hline
\end{tabular}

Fuente: elaboración propia en base a Latinobarómetro, 2018

\section{Conclusiones}

Nuestro trabajo se ha ceñido a interrogantes que, aunque no han sido explícitados a lo largo de todo el capítulo, guió el orden expositivo. Las preguntas iniciales giraron en torno a una preocupación principal: el nivel educativo y la adecuación profesional, luego de una larga estrategia individual que implica para las personas optar por la educación superior, con todos los límites que ello acarrea.

En ese sentido, la tarea calificada, que generalmente está acompañada de mejores ingresos al promedio nacional, y con protección en el empleo, está ejecutada por personas con educación superior. Sin embargo, los recursos educativos que controlan las personas en sus hogares, pertenecientes a una clase socio ocupacional de origen, no se pueden valorar con independencia de la estructura de oportunidades a la que tienen acceso.

Los estudios superiores no pueden convertirse directamente en activos en las esferas laborales en forma global, ya que previamente los límites están puestos por las estructuras de oportunidades y, en segundo lugar, por las capacidades individuales puestas en juego de tal forma para adecuarse a esos formatos laborales, que a medida que se complejizan, expulsan como atraen fuerza de trabajo.

Nuestros resultados, tomando como base principal los datos de Latinobarómetro 2018, podrían sintetizarse de la siguiente manera:

1) Existe una alta correspondencia entre educación superior y adecuación calificada laboral, un poco más del $60 \%$ de la población con estudios superiores presenta una inserción de mayor calificación. Situación que no ocurre con la población de educación media o básica.

2) Por otro lado, la inserción adecuada es relativamente similar entre varones y mujeres, aunque presenta diferencias mayores en la población de más de 55 años, 
lo cual genera otro tipo de interrogantes, si allí pesa la generación o la salida laboral de la mujer, producto de la maternidad, que le dificulta el "reenganche adecuado" en el mercado laboral. La inserción similar entre varones y mujeres jóvenes puede ser alentador en torno a las diferencias de género generales.

3) Se aprecia además que en los países andinos aumentan la adecuación hacia las edades más avanzadas, en Centroamérica y el Caribe crece la adecuación conforme lo hace también la edad de la población, y en el Cono sur se mantiene relativamente estable para los diferentes grupos etarios.

4) Y como era previsible, pero en nuestro artículo se aprecia en forma cuantitativa, las mejores recompensas materiales, están fuertemente ligadas a la educación superior en todas las regiones.

A mediano plazo, podemos plantear con un margen de error bajo, que la educación superior equilibra el tipo de inserción laboral entre géneros, se logra adecuaciones calificadas en mayor grado, y con ello mejores salarios, y recompensas de estatus. En América Latina, según la información presentada, y aún debido a la extensión de la educación, las posibilidades de experimentar mejores condiciones de vida, a pesar de los límites estructurales de origen, a esta altura del siglo, muestran un desempeño favorable sobre el desarrollo y la educación superior.

\section{Bibliografía}

BEDUWE, C. y GERME, J. F. (2004). Raising the level of education in France: from growth to stabilisation. European Journal of Education, 39 (1): 119-132

BRUNNER, J. J. (1990). Educación Superior en América Latina. Santiago de Chile: Fondo de Cultura Económica.

ECHEVERRÍA ZABALZA, J. (1999). La movilidad social en España. Madrid: Istmo.

ERIKSON, R., y GOLDTHORPE, J. H. (1992). The constant flux: A study of class mobility in industrial societies. Oxford: Oxford University Press.

CHÁVEZ MOLINA, E. (2019) ¿Trabajador Educado, Trabajo Calificado? Análisis de Niveles Educativos y Calificación del Empleo de Mujeres y Varones Activos en Argentina. En Salvia, A. (Coord.) Tendencias sobre la Desigualdad. Buenos Aires: CLACSO 
MORENO-BRID, J. C., y RUIZ-NÁPOLES, P. (2009). La educación superior y el desarrollo económico en América Latina. Serie Estudios y Perspectivas, 106, Santiago de Chile: CEPAL.

PLANAS J. y FACHELLI S. (2016). Equitat en l'accés i en la Inserció professional Dels graduats Universitaris. Agència per a la Qualitat del Sistema Universitari de Catalunya. Barcelona: AQU.

PNUD (2020). The next frontier Human development and the Anthropocene. Human Development Report 2020. New York: United Nations Development Programme

SOLÍS, P., \& DALLE, P. (2019). La pesada mochila del origen de clase. Escolaridad y movilidad intergeneracional de clase en Argentina, Chile y México. Revista Internacional de Sociología, 77(1), 118, 1-17.

RAMA, C. (2009). La tendencia a la masificación de la cobertura de la educación superior en América latina. OEI - Revista Iberoamericana de Educación. 50, 173-195.

SATRÚSTEGUI, K. U. (2009). Desarrollo, subdesarrollo, maldesarrollo y postdesarrollo: una mirada transdisciplinar sobre el debate y sus implicaciones. Bilbao: CLAES.

UNZUÉ, M. (2019). Una mirada sobre la educación superior en América Latina. Revista de Educación y Derecho. (19). 1-6

WYCZYKIER, G. (2017). La problemática del desarrollo, las clases sociales y la burguesía en América Latina: Reflexiones conceptuales. Revista Trabajo y Sociedad, No 29, Vol XVIII, 649-665 